\title{
Effects of a Self-Monitoring Quality of Life Intervention for Patients with Cancer Receiving Palliative Care in Japan: Study Protocol for a Randomized Controlled Trial
}

\author{
Ayako Matsuda ${ }^{1 *}$, Yosuke Yamada ${ }^{2}$, Noriko Ishizuka ${ }^{3}$, Eisuke Matsushima ${ }^{3}$, \\ Kunihiko Kobayashi ${ }^{4}$, Takayoshi Ohkubo ${ }^{1}$, Kazue Yamaoka ${ }^{5}$
}

\begin{abstract}
Background: Previous studies suggest the use of patient-reported outcome measures in routine clinical practice has important benefits for patients with cancer, particularly as feedback regarding patients' quality of life (QOL) improves doctor-patient communication and clinical decision making. This study aimed to examine the effect of using the Care Notebook as a routine self-monitoring QOL intervention in clinical practice for patients with cancer receiving palliative care. The results are expected to clarify the practical use of the Care Notebook in this population. Methods: This prospective randomized study is being undertaken at Toshima Hospital, Japan. Participating patients who are randomly assigned to the intervention group will be asked to complete the shortened Care Notebook booklet for patients with cancer in palliative care once each day. A control group will receive usual care. The primary outcome is global health status/QOL (Global QOL), as assessed by the European Organization for Research and Treatment of Cancer Quality of Life Questionnaire Core 15 Palliative. Data will be collected at baseline (after allocation), and at 1 week and 3 weeks in both the control and intervention groups. The effects of the intervention will be evaluated with a mixed random effects model. The required sample size is 200 patients. We obtained approval from Toshima Hospital (No 26-11) and the Tokyo Medical and Dental University Ethics Committee (No 1756). The findings will be disseminated through publications in peer-reviewed journals and attendance at domestic and international conferences. The trial was registered with the UMIN clinical trials registry (Trial registration number: UMIN000025322). Conclusions: This study will provide evidence on whether medical staff can use the Care Notebook as a routine self-monitoring QOL intervention in clinical practice for patients with cancer receiving palliative care. We expect that a routine Care Notebook intervention for patients with cancer will be recommended in healthcare facilities.
\end{abstract}

Keywords: Self monitoring- quality of life- cancer- palliative care

Asian Pac J Cancer Prev, 19 (11), 3027-3032

\section{Introduction}

Subjective assessments including quality of life (QOL) are just as important for patients with cancer as objective assessments such as survival and response rates (Matsuda et al., 2014). QOL assessment is often used as an outcome measure in clinical trials. A future task in this context is how QOL assessment information is fed back to patients. Cancer diagnosis and treatments are known to affect patients' QOL. However, medical staff may not sufficiently understand patients' problems and difficulties, including QOL. Previous studies suggested that the use of patient-reported outcome measures in routine clinical practice has important benefits for patients with cancer, and feedback regarding QOL information improves doctor-patient communication and clinical decision making (Rubenstein et al., 1995; Espallargues et al., 2000; Detmar et al., 2002; Gilbody et al., 2002; Velikova et al., 2004; Greenhalgh et al., 2005; Boyes et al., 2006; Valderas et al., 2008; Fischer et al.,2012). However, these effects are not clear among patients with cancer who are receiving palliative care.

To measure patients' QOL in daily clinical oncology practice, Kobayashi and colleagues developed the Care Notebook, and examined its validity and reliability (Kobayashi et al., 2005). The Care Notebook allows clinical oncologists to easily and repeatedly collect QOL information on physical, mental, and life wellbeing with minimal patient burden (Kobayashi et al., 2005). The Medical Oncology Department of Leiden University Medical Centre in the Netherlands developed a self-monitored QOL intervention for routine clinical 
practice based on the Care Notebook. However, the effects of using the Care Notebook in clinical practice have not yet been examined among patients receiving palliative care. In Japan Society of Gynecologic Palliative Medicine (JSGPM) Survey, as Futagami et al., (2016) reported in the results of this survey, indicated that the systems providing end-of-life care for patients with incurable cancer were not sufficiently established in Japan.

The primary objective of this study is to examine the effects of the Care Notebook as a routine self-monitoring QOL intervention to improve patient-reported global QOL in patients with cancer receiving palliative care. The secondary objectives are to evaluate the relationships between global QOL and QOL subscales, psychological characteristics, and sociodemographic and clinical variables. Our study is expected to clarify the practical use of the Care Notebook for patients with cancer receiving palliative care.

\section{Materials and Methods}

\section{Study design}

The trial design follows the Standard Protocol Items: Recommendations for Interventional Trials (SPIRIT) 2013 statement (Chan et al., 2013).

This prospective randomized study started at Toshima Hospital in May 2015, and will conclude in March 2019. All participants will be asked to provide written informed consent to participate in the study. After completion of consent and a case report form (CRF) by researchers, patients will be randomized to the intervention group or the control group (usual care) (Figure 1).

The CRF covers items such as date of birth, age, sex, diagnostic names, Eastern Cooperative Oncology Group performance status (PS), first hospitalized day, cancer stage, previous history, complications, end day of aggressive therapy, treatment details (surgery (yes/ no), radiotherapy (yes/no), chemotherapy (yes/no)), and date of death.

\section{Study setting}

After obtaining approval from Toshima Hospital and the Tokyo Medical and Dental University Ethics Committee, the trial was registered with the UMIN clinical trials registry (Trial registration number: UMIN000025322. Issue Date: 31 May 2017).

Patients who receive palliative care at Toshima Hospital are first hospitalized. If patients are able to perform home care, they return home and visit the hospital as an outpatient. Recruitment is set at the time of first hospitalization.

\section{Patients and eligibility criteria}

Eligibility criteria include patients aged 20 years or more who are in a physical condition that means they can tolerate the investigation. Detailed inclusion and exclusion criteria are shown in Table 1.

\section{Randomization and blinding}

Eligible participants are randomly assigned using a permuted-block technique with a randomization list (random permutated blocks with a block size of four) (Hulley et al., 2001). Allocation to the intervetion group is performed by the principal reseacher. Eligible patients and reseachers are not informed to which group patients are randomized, but no blinding is performed.

\section{Intervention \\ Intervention group}

Patients randomly assigned to the intervention group are asked to complete the Care Notebook booklet questionnaire once each day in addition to usual care. To evaluate the Care Notebook intervention, patients will complete the Care Notebook checklist at 1 week and 3 weeks. Care Notebook checklist questions include: "Was the Care Notebook helpful?"; "How about the contents?"; "How was the quantity?"; "How about the timing when the Care Notebook booklet was distributed?"; and "Please fill in the sheet if you would like to tell us your opinion and suggestions."

The overall aim of the intervention (Care Notebook booklet) is to inform a communication system between patients and medical staff. To promote communication, medical staff needs to understand the patient's status and patients need to understand their condition, including QOL. We used the Care Notebook to achieve this. The Care Notebook (Care Notebook Center: http://www. care-notebook.com/en/download.html) which has been validated and reported, was designed to assess QOL among patients with cancer. We used a shortened questionnaire (Care Notebook) for patients with cancer in palliative care. The Care Notebook is a self-administered, cancer-specific questionnaire that asks about patients' conditions using 14 items structured in multidimensional scales. The questionnaire consists of three major scales: physical wellbeing (P1-P10), mental wellbeing (M2, M4), and life wellbeing (L7, L8). These scales are divided into several subscales. Physical wellbeing has three multi-item subscales: appetite loss (P3, P4, P7), constipation (P6, $\mathrm{P} 8)$, and fatigue (P9, P10); three single-item measures (pain, P1; shortness of breath, P2; and sleeping trouble, P5); and also measures subjective QOL (L7, L8). Each item uses one word or a short phrase and responses are on an 11-point linear analog scale (0-10). A score of 10 in physical and mental wellbeing indicates the heaviest burden. A score of 10 in life wellbeing indicates the best possible function or QOL; therefore, the polarity of life wellbeing data was reversed before analysis so that a higher score indicated poorer QOL in all questionnaire items. The Care Notebook questionnaires for the 3 weeks were bundled in a booklet form. In this study, the Care Notebook is used as a self-monitoring QOL intervention for routine clinical practice. The Care Notebook booklet can also be used as a diary to support communication between patients and medical staff.

\section{Control group}

The control group received "usual care" or routine practice that medical staff provides during course of routine clinical care for patients with cancer receiving palliative care. 


\section{Study hypothesis}

The hypothesis underlying this study is that patients with cancer who are receiving palliative care and complete the Care Notebook booklet (intervention group) will have a better Global QOL than those who receive only usual care (control group).

\section{Outcome measures}

\section{Primary outcome}

The primary outcome is global health status/QOL (Global QOL) as reported by patients, assessed by using the European Organization for Research and Treatment of Cancer Quality of Life Questionnaire Core 15 Palliative (EORTC QLQ-C-15 PAL) (Groenvold, et al. 2006; Echteld, et al., 2006; Groenvold, et al., 2006). A Global QOL score of 100 indicates the best possible QOL.

\section{Secondary outcomes}

1. Physical and emotional function, and symptom items, as measured by the EORTC QLQ-C15-PAL. The EORTC QLQ-C15-PAL contains 15 items that are identified as important in the palliative population. Patients rate physical function, emotional function, and symptom items (pain, fatigue, nausea/vomiting, appetite, dyspnea, constipation, and sleep) in addition to Global QOL. A score of 100 in physical and mental wellbeing indicates the best possible function or QOL. A score of 100 on the symptoms scale indicates the heaviest burden.

2. QOL was measured by the Functional Assessment of Chronic Illness Therapy-Palliative Care (FACIT-PAL). The FACIT system provides a general, multidimensional measure of health-related QOL (FACT-G) (Cella, et al., 1993) including a 27-item general measure that can be augmented with disease or symptom-specific subscales. The FACT-G captures four health-related QOL domains: physical, social, emotional, and functional wellbeing. The 19-item FACIT-PAL palliative care subscale has undergone little psychometric evaluation to date (Lyons et al., 2009).

3. Anxiety and depression, as measured by the Hospital Anxiety and Depression Scale (HADS) (Bjelland et al., 2002). The HADS performs well in assessing symptom severity and caseness of anxiety disorders and depression in somatic, psychiatric, and primary care patients, and in the general population. It is divided into anxiety and depression subscales, each containing seven

Table 1. Inclusion and Exclusion Criteria

\begin{tabular}{lc}
\hline Inclusion criteria & Exclusion criterion \\
\hline Age $\geq 20$ years & \\
Patients diagnosed with cancer and & \\
received a notice from their doctor & \\
about cancer. & Patient participation is \\
Curative treatment discontinued or & considered inappropriate \\
little expected benefit in terms of & by based on their doctor's \\
overall survival & judgement. \\
PS 0 -3 & \\
Patients have prediction beyond 1 & \\
month. & \\
\hline 1, PS, Eastern Co-operative Oncology Group (ECOG) performance \\
status
\end{tabular}

intermingled items.

4. Global Rating of Change scales (GRCS) (Juniper, et al., 1994). The GRCS is a standardized, symmetric scale on which patients specify their level of agreement/ disagreement varying between -7 and +7 that reflects their perception of the change over time in their symptoms.

\section{Data collection and time points}

Sociodemographic data for eligible participants will be collected at baseline (after allocation). Primary and secondary outcomes will be collected at baseline (after allocation), and at 1 week and 3 weeks in both the control and intervention groups (Figure 1).

\section{Participant characteristics}

Sociodemographic data include sex, age, occupation, education, marital status, and children (yes/no). Clinical data (diagnostic names, PS, first hospitalized day, cancer stage, previous history, complication, end day of aggressive therapy, treatment details (surgery, radiotherapy, chemotherapy), and date of death) will be obtained from the CRF.

\section{Sample size calculation}

The sample size required for the study was calculated assuming an effect size of 0.56 (mean change score 19.1, standard deviation 34.2). The mean change score was mean change score for physical functioning from baseline (month 1 - baseline) in patients with deteriorated QOL as defined by an overall QOL anchor (Bedard et al., 2016). Using a two-sided significance level of $5 \%$ and a power of $90 \%$, and assuming a dropout rate of $30 \%$ (Arraras et al., 2014), a sample size of 200 was estimated to be sufficient.

\section{Results}

\section{Statistical analysis}

The primary outcome will be analyzed following the intention to treat (ITT) principle. For ITT, the last observation carried forward method will be used to manage missing data due to attrition. Further missing outcomes will be analyzed using ignorable maximum likelihood.

In per protocol set (PPS), patients with poor compliance to the study protocol and who refuse to receive the allocated Care Notebook booklet after baseline will be removed from the analysis. In addition, if a patient has not completed the Care Notebook booklet more than half of the time at 1 week, we will consider that patient as not able to complete the intervention and they will be excluded from PPS analysis. We will also conduct PPS analysis, including all randomized participants, where outcome data are available.

A mixed effects model will be used to compare the effects of the intervention on the primary and secondary outcomes. We also plan to conduct subgroup analyses according to patient characteristics using the HADS, GRCS, and sociodemographic data. $\mathrm{P}<0.05$ (two-sided) will be considered statistically significant. Statistical analyses will be performed with SAS Ver.9.4. 


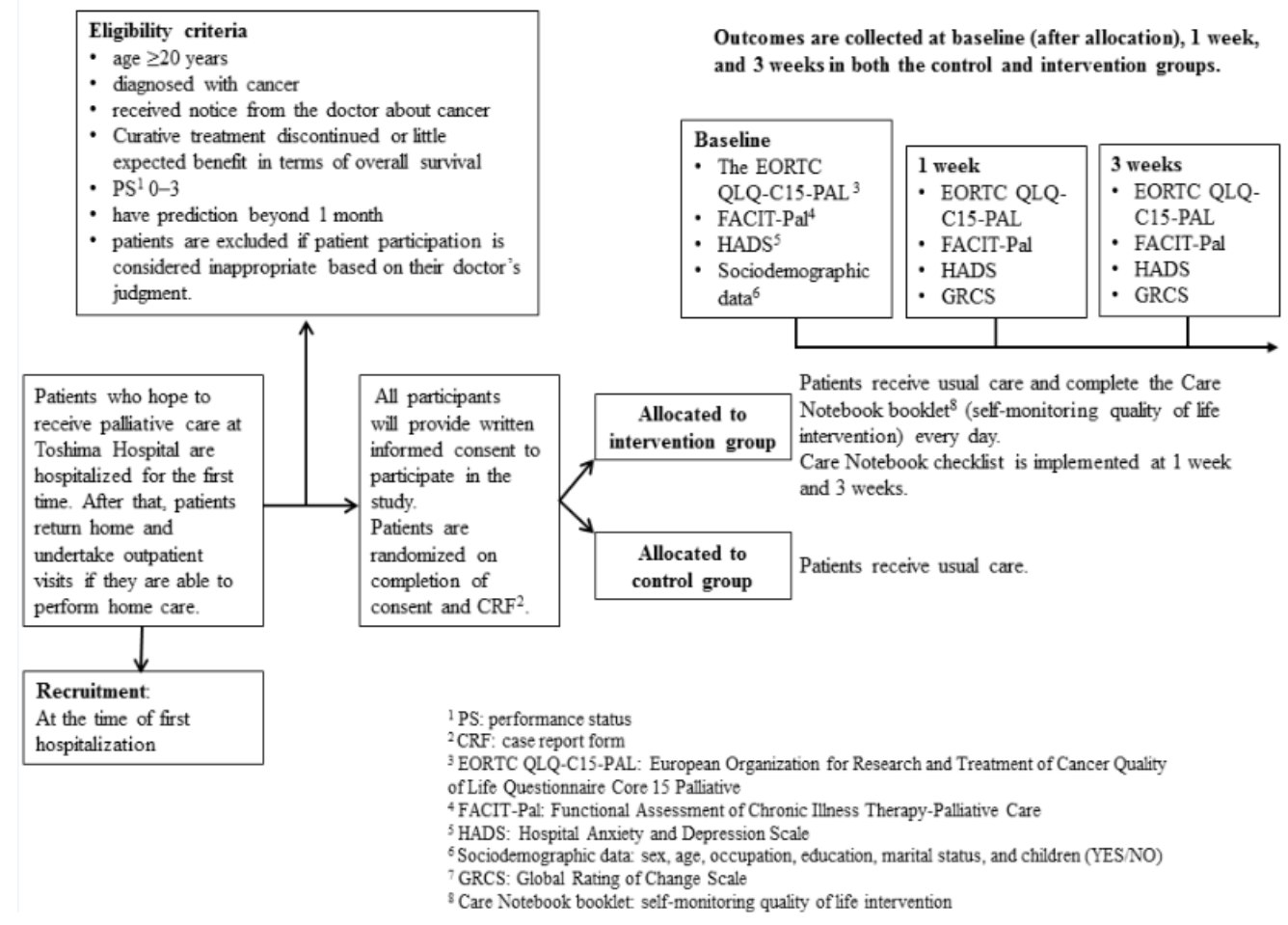

Figure 1. The Study Flow

\section{Data management}

Personal information obtained in this study will be coded. Participant files will be stored in numerical order in a secure but accessible place and manner. Data will be anonymized before analysis, and kept in a password-protected computer with limited access. The study organizer will keep all documents related to this trial in a locked cabinet for 10 years at the Tokyo Medical and Dental University, Graduate School of Medical and Dental Sciences. Five years after conclusion of the study or 3 years after the final publication of the results (whichever is later), all documents will be disposed of using the document destruction service contracted by the University.

\section{Conditions for discontinuation of this study and actions in that event}

When significant information about safety or effectiveness of the intervention is obtained, the study organizer will determine whether to continue the study.

\section{Monitoring}

Information on when the study begins, the conduct of the study (sample size), ethical considerations, occurrence of any detrimental or adverse events, study results, and registration of the study with a public database will be submitted to the relevant Ethics Committee in annual interim reports. A report will also be submitted to the Ethics Committee on conclusion of the study when the final results are presented.

\section{Protocol amendments}

If any amendments are made, the Ethics Committee will be notified as necessary.

\section{Ethics and dissemination}

This trial has been approved by Toshima Hospital (No 26-11) and the Tokyo Medical and Dental University Ethics Committee (No 1756). All eligible patients will be provided with detailed written information and have opportunity to discuss participation with medical doctors or researchers before providing written informed consent. Patients without capacity to consent will be excluded from the trial. The findings will be disseminated through publications in peer-reviewed journals and attendance at national and international conferences.

\section{Discussion}

QOL assessments are often been for research purposes, although QOL monitoring should also be implemented in daily clinical routines. In some studied, the survey about the palliative care education and training program was carried out (Nagamatsu Y, et al., 2014; Ratanakaaew, et al., 2015). We consider that the Care Notebook can be included in daily clinical routines as the palliative care program. This study will provide evidence on whether medical staff can use the Care Notebook as a routine self-monitoring QOL intervention in clinical practice for patients with cancer receiving palliative care. The possible benefits of a routine Care Notebook intervention for patients with cancer receiving palliative care can be summarized as:

1. To enable medical staff to capture patient status (including QOL) correctly and patients to check the status of their own QOL.

2. To provide a medical communication system to support communication between patients and medical staff.

3. To provide a medical support system capable 
of resolving patients' problems through collaboration between patients and medical staff.

4. To enable medical staff to provide a variety of information to patients and facilitate discussion of this information between patients and medical staff.

As Erharter et al., (2010) reported in the previous study, indicated that computer-based QOL monitoring was useful for early detection of physical symptoms and psychosocial problems. In a further study, we should consider the practical use of the Care Notebook. We expect that a routine Care Notebook intervention for patients with cancer will be recommended in healthcare facilities for palliative care.

Finally, there are limitations in this study. This study does not use a double-blind design, but eligible patients and researchers will not be informed to which group patients are randomized until the study starts. In addition, the outcome measures have been clearly set and use reliable scales and the test is adequately powered to detect effects on the outcomes.

\section{Authors' contributions}

AM conceived the study. AM and KY participated the development of the protocol. YY, NI, and EM were involved in trial design, intervention development and ethics application. AM and KY will be responsible for statistical analyses. AM and KY wrote the first draft of this paper. All authors contributed to preparing and revising the manuscript, including relevant scientific content. All authors approved the final version of the manuscript.

\section{Competing interests}

None declared.

\section{Ethics approval}

Toshima Hospital (No 26-11) and the Medical Research Ethics Committee of Tokyo Medical and Dental University (No 1756).

\section{Provenance and peer review}

Not commissioned; externally peer reviewed.

\section{Data sharing statement}

The informed consent documents are available on request.

\section{Acknowledgements}

This study is financially supported by the Ministry of Education, Culture, Sports, Science and Technology in Japan Grant-in-Aid for Scientific Research Grant B in 2015 (Grant No.15K19295).

\section{References}

Arraras JI, de la Vega FA, Asin G, et al (2014). The EORTC QLQ-C15-PAL questionnaire, validation study for Spanish bone metastases patients. Qual Life Res, 23, 849-55.

Bedard G, Zeng L, Zhang L, et al (2016). Minimal important differences in the EORTC QLQ-C15-PAL to determine meaningful change in palliative advanced cancer patients.
Asia Pac J Clin Oncol, 12, 38-46.

Bjelland I, Dahl AA, Haug TT, Neckelmann D (2002). The validity of the hospital anxiety and depression scale. An updated literature review. J Psychosom Res, 52, 69-77.

Boyes A, Newell S, Girgis A, McElduff P, Sanson-Fisher R (2006). Does routine assessment and real-time feedback improve cancer patients' psychosocial well-being?. Eur J Cancer Care, 15, 163-71.

Care Notebook Center. http://www.care-notebook.com/en/ download.html (accessed July 28, 2017).

Cella DF, Tulsky DS, Gray G, et al (1993). The functional assessment of cancer therapy scale, development and validation of the general measure. J Clin Oncol, 11, 570-9.

Chan AW, Tetzlaff JM, Altman DG, et al (2013). SPIRIT 2013 statement, defining standard protocol items for clinical trials. Ann Intern Med, 158, 200-7.

Detmar SB, Muller MJ, Schornagel JH, Wever LD, Aaronson NK (2002). Health-related quality-of-life assessments and patient-physician communication, a randomized controlled trial. JAMA, 288, 3027-34.

Echteld MA, Deliens L, Onwuteaka-Philipsen B, Klein M, van der Wal G (2006). EORTC QLQ-C15-PAL, the new standard in the assessment of health-related quality of life in advanced cancer?. Palliat Med, 20, 1-2.

Erharter A, Giesinger J, Kemmler G, et al (2010). Implementation of computer-based quality-of-life monitoring in brain tumor outpatients in routine clinical practice. J Pain Symptom Manage, 39, 219-29.

Espallargues M, Valderas JM, Alonso J (2000). Provision of feedback on perceived health status to health care professionals, a systematic review of its impact. Med Care, 38, $175-86$.

Fischer MJ, Krol-Warmerdam EM, Ranke GM, et al (2012). Routine monitoring of quality of life for patients with breast cancer, an acceptability and field test. J Psychosoc Oncol, 30, 239-59.

Futagami M, Yokoyama Y, Sato T, et al (2016). Palliative care for patients with gynecologic cancer in Japan: A Japan society of gynecologic palliative medicine (JSGPM) survey. Asian Pac J Cancer Prev, 17, 4637- 42.

Gilbody SM, House AO, Sheldon T (2002). Routine administration of health related quality of life (HRQoL) and needs assessment instruments to improve psychological outcome-a systematic review. Psychol Med, 32, 1345-56.

Greenhalgh J, Long AF, Flynn R (2005). The use of patient reported outcome measures in routine clinical practice, lack of impact or lack of theory?. Soc Sci Med, 60, 833-43.

Juniper EF, Guyatt GH, Willan A, Griffith LE (1994). Determining a minimal important change in a disease-specific Quality of Life Questionnaire. J Clin Epidemiol, 47, 81-7.

Groenvold M, Petersen MA, Aaronson NK, et al (2006). EORTC QLQ-C15-PAL, the new standard in the assessment of health-related quality of life in advanced cancer?. Palliat Med, 20, 59-61.

Groenvold M, Petersen MA, Aaronson NK, et al (2006). The development of the EORTC QLQ-C15-PAL, a shortened questionnaire for cancer patients in palliative care. Eur $J$ Cancer, 42, 55-64.

Kobayashi K, Green J, Shimonagayoshi M, et al (2005). Validation of the care notebook for measuring physical, mental and life well-being of patients with cancer. Qual Life Res, 14, 1035-43.

Lyons KD, Bakitas M, Hegel MT, et al (2009). Reliability and validity of the functional assessment of chronic illness therapy-palliative care (FACIT-Pal) scale. J Pain Symptom Manage, 37, 23-32.

Matsuda A, Yamaoka K, Tango T, Matsuda T, Nishimoto 
H (2014). Effectiveness of psychoeducational support on quality of life in early-stage breast cancer patients, a systematic review and meta-analysis of randomized controlled trials. Qual Life Res, 23, 21-30.

Nagamatsu Y, Nakayama Y, Clayson H, et al (2014). Caring for patients with malignant pleural mesothelioma in Japan: evaluation of a Palliative Care Educational Program. Asian Pac J Cancer Prev, 15, 9165-70.

Ratanakaaew A, Khemapech N, Laurujisawat P (2015). Palliative care education in gynecologic oncology: a survey of gynecologic oncologists and gynecologic oncology fellows in Thailand. Asian Pac J Cancer Prev, 16, 6331-4.

Rubenstein LV, McCoy JM, Cope DW, et al (1995). Improving patient quality of life with feedback to physicians about functional status. J Gen Intern Med, 10, 607-14.

Stephen BH, Steven RC, Warren SB, et al (2001). Designing clinical research. in 'designing an experiment: clinical trials 1', lippincott williams and wilkins, 530 Walnut street Philadelphia, PA 19106 USA, pp 148-9.

Valderas JM, Kotzeva A, Espallargues M, et al (2008). The impact of measuring patient-reported outcomes in clinical practice, a systematic review of the literature. Qual Life Res, 17, 179-93.

Velikova G, Booth L, Smith AB, et al (2004). Measuring quality of life in routine oncology practice improves communication and patient well-being, a randomized controlled trial. JClin Oncol, 22, 714-24.

This work is licensed under a Creative Commons AttributionNon Commercial 4.0 International License. 